\title{
Flow Dynamics Control The Location of Sprouting and Direct Elongation During Developmental Angiogenesis
}

Siavash Ghaffari ${ }^{1,2}$, Richard L. Leask ${ }^{2}$, Elizabeth A.V. Jones ${ }^{1,2,3}$ *

${ }^{I}$ Lady Davis Institute for Medical Research, McGill University, 3755 Ch. Côte-Ste-Catherine, Montréal, QC, H3T 1E2, Canada.

${ }^{2}$ Department of Chemical Engineering, McGill University, 3610 University St., Montréal, QC, H3A 0C5, Canada

${ }^{3}$ Department of Cardiovascular Science, KU Leuven, UZ Herestraat 49 - box 911, 3000 Leuven, Belgium

To whom correspondences should be addressed: Elizabeth A.V. Jones, Centre for Molecular and Vascular Biology, KU Leuven, UZ Herestraat 49 - box 911, 3000 Leuven, Belgium, Tel. +32 163475 58; Fax.+32 163460 01; Email: liz.jones@med.kuleuven.be

Short Title: Hemodynamic during angiogenesis 


\begin{abstract}
Angiogenesis is tightly controlled by a number of signalling pathways. Though our understanding of the molecular mechanisms involved in angiogenesis has rapidly increased, the role that biomechanical signals play in this process is understudied. We recently developed a technique to simultaneously analyse flow dynamics and vascular remodelling by time-lapse microscopy in the capillary plexus of avian embryos and used this to study the hemodynamic environment present during angiogenic sprouting. We found that sprouts always form from a vessel at lower pressure towards a vessel at higher pressure. We found that sprouts form at the location of a shear stress minimum, but avoid locations where two blood streams merge even if this point is at a lower level of shear stress than the sprouting location. Using these parameters, we were able to successfully predict sprout location in embryos. We also find that the pressure difference between two vessels is permissive to elongation, and that sprouts will either change direction or regress if the pressure difference becomes negative. Furthermore, the sprout elongation rate is proportional to the pressure difference between the two vessels. Our results show that flow dynamics are predictive of the location of sprout formation in perfused vascular networks and that pressure differences across the interstitium can guide sprout elongation.
\end{abstract}

Keywords: Angiogenesis, Hemodynamics, Time-lapse microscopy, micro-Particle image velocimetry, Computational fluid dynamics 


\section{Introduction}

The vasculature is the first organ to form during development and it adapts to the onset of blood flow in part by forming new blood vessels through angiogenesis. The ability to control angiogenesis has important therapeutic applications for many diseases. Anti-angiogenic treatments used to treat tumours lead to transient vessel normalization, which causes a reduction in tumour invasiveness and better delivery of chemotherapeutic drugs (Carmeliet and Jain, 2011). In wet macular degeneration, defective angiogenesis leads to the formation of leaky vessels that damage the eye and lead to vision loss. Anti-angiogenic therapies can effectively stop and sometimes reverse the progression of macular degeneration (Tah et al., 2015). As such, there is significant interest in understanding the signals that induce or inhibit angiogenesis.

In the adult vasculature, endothelial cells exist in a quiescent state whereby little proliferation is present (Hanahan and Folkman, 1996; Hobson and Denekamp, 1984). Normal physiological levels of shear stress maintains endothelial cells in a quiescent state by inducing cell cycle arrest (Lin et al., 2000) and strengthening tight junctions (Lin et al., 2000). For sprouting to occur, however, endothelial cells must re-enter the cell cycle (Bai et al., 2014), loosen their attachment to neighbouring cells (Lampugnani and Dejana, 2007), degrade the local basement membrane (Siefert and Sarkar, 2012) and then invade the tissue to form a new vessel. Though shear stress is known to affect many of these pathways, very little is known about the effects of shear stress on angiogenesis. Two groups recently developed micromachined flow cells that enabled them to alter shear stress and interstitial flow as endothelial cells sprout (Galie et al., 2014; Song and Munn, 2011). Both groups found that interstitial flow enhanced angiogenesis, however one found that shear stress inhibited sprouting whereas the other found that it induced sprouting (Galie et al., 2014; Song and Munn, 2011). Using an embryonic model, our group has demonstrated that increasing blood viscosity, which increases shear stress, results in a decrease in vascular density (Chouinard-Pelletier et al., 2013). We showed by time-lapse microscopy that this occurs because of a decrease in 
sprouting angiogenesis. Changing viscosity, however, also altered other hemodynamic parameters such as the pressure drop through the network. These studies establish a role for flow dynamics in angiogenesis but a better understanding of the hemodynamic environment present in vivo during sprouting is needed to identify the causal stimuli.

The vascular network must not only deliver oxygen and nutrients to tissues, but it must also be hemodynamically efficient. If a new vessel forms in hemodynamically inefficient location, then a high resistance to flow will be present and limited flow will pass through that vessel. The production of a hemodynamically efficient vascular network can occur either by excessive production of blood vessels followed by the pruning of inefficient vessel, or by sprouting of new vessels in hemodynamically efficient locations in the first place. Though vascular networks do overproduce vessels and prune inefficient ones (Pries and Secomb, 2014), this does not negate the possibility of a bias in the site of sprout initiation in perfused vascular networks. It therefore makes physiological sense that the forces created by blood flow could influence the angiogenic process.

\section{Results}

\section{Sprout Location Can Be Predicted Based on Flow Dynamics}

To study the role that flow dynamics play in the process of angiogenesis, we used a technique to simultaneously image vessel morphology and flow dynamics by time-lapse microscopy in the capillary plexus of avian embryos undergoing vascular remodelling (Ghaffari, et al. Development, accepted). We injected AlexaFluor 488-labeled acetylated low-density lipoprotein (AF488-acLDL), which specifically labels endothelial cells and macrophages (Brown et al., 1980; Hallmann et al., 1987). AF488-AcLDL labels not only perfused vessels, but also sprouting endothelial cells. We then injected red fluorescent microspheres to follow blood flow dynamics and imaged the microspheres with a high-speed camera. We analysed the pattern of blood flow present during sprouting in the arterial plexus of three quail embryos 
as well as the venous plexus of three additional quail embryos, for a total of 6 time-lapse recordings (Fig. 1A). Early in vascular development, a spatial separation exists such that the rostral capillary plexus is venous and the caudal plexus is arterial. The motion of the microspheres was analysed using a technique called micro-particle image velocimetry ( $\mu \mathrm{PIV})$ to calculate the velocity of the blood. Micro-PIV was only used to make velocity measurements in straight vessel segments that led in and out of a region of interest. These measurements were then used as the input for a computational fluid dynamic solver to calculate flow in the entire region of interest, around the growing sprout. Flow dynamics were analysed starting 30 minutes before the sprout was visible (yellow arrowheads) and continued until the new vessel lumenized and carried flow (Fig. 1B). The computational solver calculates blood velocity in the entire region of interest from the micro-PIV inputs (Fig. 1CD), as well as calculating shear stress, pressure drop and vorticity (Supplemental Movie 1-3).

We first investigated the relationship between flow parameters and the location of sprout initiation. We found that sprouts consistently formed from vessels at lower pressure towards vessel at higher pressure ( $n=6$ of 6 embryos, $p=0.014$, Fig. 2 ). The contour plots of relative pressure 30 minutes before sprout formation are presented, both for the arterial regions of the plexus (Fig. 2A) and the venous regions (Fig. 2C). The location and direction of sprouting are identified by the black arrows (Fig. 2A, C) as observed in the green images of vascular morphology after the sprout has formed (Fig. 2B, D, yellow arrowheads). The average pressure difference between the initiation site and the opposite vessel (i.e. the one towards which the sprout is extended) was of the same order of magnitude in all embryos. Pressure differences were normalized by the distance between the two vessels. The average value was $+2.1 \mathrm{~Pa} / \mathrm{mm}$ (standard error $=0.09, \mathrm{n}=3$ ) for arteries and $+1.7 \mathrm{~Pa} / \mathrm{mm}$ (standard error $=0.09$, $\mathrm{n}=3$ ) for veins. Though sprouts formed from lower towards higher pressure vessels, the location and direction of sprouting was not at the maximum pressure difference in any of the time-lapse experiments. 
Shear stress has been shown to play a significant role in controlling angiogenesis. We therefore investigated the level of shear stress on the endothelium when sprouting initiated (i.e. 30 minutes prior to sprout appearance, Fig. 3). Shear stress contour plots just prior to sprouting for the three arterial embryos are shown in Figure 3A. We plotted the level of shear stress with respect to distance along the endothelium (Fig. 3B). The black lines and arrows in Figure $3 \mathrm{~A}$ indicate the arc length represented in the $\mathrm{x}$-axis of Figure $3 \mathrm{~B}(\mathrm{x}=0$ at the bottom of the arrow). Shear stress levels were plotted for a subsection of the lower pressure region, however plots of the entire region are available in Supplemental Figure 1. We found that for all arterial time-lapse recordings, the location of sprouting can be identified by a local minimum in shear stress (sprouting location indicated by black arrows, $n=3$ of 3 embryos, Fig. 3B). Our previous results had indicated that shear stress inhibited sprouting angiogenesis in the venous but not the arterial plexus (Chouinard-Pelletier et al., 2013). In our venous timelapse experiments, the sprout location was not associated with any specific pattern or level of shear stress (data not shown). One occurred at a minimum; the second at a midpoint; and the third was very close to the point with highest level of shear stress in the region. Therefore, in veins, our current results do not support a correlation between the level of shear stress and the sprouting location.

Though sprouts in the arterial plexus formed at a local minimum in shear stress, this was not always the absolute minimum. We investigated flow patterns at all shear stress minimum (Fig. 4A, blue arrow represents sprouting location, orange arrow represents the second shear stress minimum in Embryo 1). We observed that non-sprouting shear stress minima occurred in regions where two streams of blood flow merged (Fig. 4A, n=3 of 3 embryos). Vorticity is the angular velocity of a fluid. Convergence or divergence of streams results in a sign change in the vorticity. Regions where streams converge can clearly be identified in the contour plots of the vorticity because the vorticity changes from a positive to a negative value (Fig. 4B, orange arrow indicating region with a change from red to blue). Though there is significant vorticity in this region, this occurs because of the angular velocity of the fluid that results 
from the streams converging and not because of the presence of recirculation. We plotted shear stress (Fig. 4C, E, G) and vorticity (Fig. 4D, F, H) for the entire region at lower pressure. If points where the vorticity passes through zero are excluded from the analysis, then the lowest minimum in shear stress defines the location of sprouting ( $\mathrm{n}=3$ of 3 embryos, $\mathrm{p}=0.010)$

We next tested whether we could use these observations to predict sprouting location. We analysed flow dynamics prior to sprouting in the arterial region of 4 new embryos (Fig. 5). The region and time point that were analysed were chosen by one author but analysed by a different author, making the analysis blind to sprouting location. Sprouting location was restricted to the bottom $50 \%$ of pressure values (Fig. $5 \mathrm{~A}$ ). Within the lower pressure region, we identified all shear stress minima and all locations where vorticity equalled zero (Fig. 5B). Excluding locations with zero vorticity, the lowest minimum was chosen as the predicted sprout location (Fig. 5B). The actual sprout location was then assessed (Fig. 5C), blind to predicted sprout location, based on the green images of vascular morphology and the location of $x=0$ from the pressure contour plots. In all embryos $(n=4$ of 4 embryos, $p=0.001)$, we were able to correctly predict sprout location.

Sprout Elongation Rate is Proportional to the Pressure Difference Between the Two Vessels We frequently observed that sprouts would extend and retract before finally connecting to form a lumenized vessels (Fig. 6A, yellow arrows indicate direction of sprout movement), as has been observed in other model systems (Murakami et al., 2006). We plotted the elongation rate of the sprouts (Fig. 6B), measured by the displacement of the tip of the sprout between two frames, and compared this to changes in the three analysed hemodynamic parameters in our system. We observed that regression of the sprout occurred when the difference in pressure between the vessels became negative (Fig. $6 \mathrm{C}, \mathrm{n}=3$ of 3 embryos). We therefore plotted the elongation rate with respect to the pressure difference between the two vessels (Fig. 6D). Negative elongation rates represent sprout regression. We found a logarithmic 
relationship between the elongation rate and the pressure difference between the vessels (Fig. 6E). The non-linear regression gave an $\mathrm{r}^{2}$ value of 0.82 (Fig. 6D), whereas the linear regression on the semi-ln plot gave an $\mathrm{r}^{2}$ value of 0.87 (Fig. $6 \mathrm{E}, \mathrm{p}<0.05$ ). Thus, these results show that there is a significant association between the rate of elongation and the pressure difference between two vessels.

In some time-lapse experiments, we observed the direction of elongation changing dynamically in relation to pressure differences between two vessels. This occurred in 2 of the 3 arterial time-lapse recordings. In one example, taken from Embryo 2 of the arterial timelapse recordings (Fig. 7), the sprout was extending at time point 210 minutes (Fig. 7A). The middle panels show the contour maps for relative pressure in this region with the extending sprout labelled in black (Fig. 7B). At 210 minutes, the pressure difference between the vessels in the sprouting direction was positive (Fig. 7C). The flow pattern changed, resulting in a negative pressure difference in the direction of sprouting by 240 minutes. The sprout retracted slightly and then changed direction towards a point that resulted in a positive pressure difference ( $\mathrm{t}=270$ minutes, Fig. $7 \mathrm{C}$ ). Sprouts did not necessarily extend along the maximum pressure difference. They would continue in the same direction as long as the pressure difference was positive but changed direction or regressed when the pressure difference was negative. As such, the pressure difference was permissive rather than instructive.

\section{Discussion}

We show that the flow dynamics have a significant effect on the sprouting location, the direction of sprouting and the rate of elongation. We found that sprouts only form from a vessel at low pressure towards a vessel at higher pressure, such that there is a positive pressure difference at the sprouting location. Within the lower pressure region, sprouts formed at the lowest minimum in shear stress once locations where streams merge were excluded. 
Our results do not, however, show that fluid dynamics control when a sprout will form, but rather where they are likely to form. Previous in vitro work on sprouting and shear stress had shown that shear stress could inhibit VEGF-induced sprouting (Song and Munn, 2011). It is important to note that in that system, without the VEGF, no sprouting was observed. Similarly, in our system, fluid dynamics did not predict which avascular regions would sprout, but instead the flow predicted where along the vessel wall sprouting occurred. We did find that the sprouting avascular regions were larger on average than non-sprouting avascular regions ( $\mathrm{n}=10$ for each, $\mathrm{p}=0.012$, Supplementary Fig. $2 \mathrm{~A}$ ). Given the observed spread within the data (Supplementary Fig. 2B), the size of the avascular region alone cannot predict which regions will sprout. Our results suggest that the formation of a sprout within an avascular region is initiated by non-hemodynamic parameters, but that the fluid dynamic parameters define the position of that sprout.

Our results support that shear stress is inhibitory to sprouting, as we and others have found (Chouinard-Pelletier et al., 2013; Song and Munn, 2011; Tressel et al., 2007). More recently, another group published that that shear stress induced rather than inhibited sprouting (Galie et al., 2014). It is possible that both extremes are permissive to sprouting. Both low $(<10$ $\mathrm{dyn} / \mathrm{cm} 2)$ and pathologically high shear stress $(>50 \mathrm{dyn} / \mathrm{cm} 2)$ result in increased cell turnover by stimulating both apoptosis (Davies et al., 1986; Dolan et al., 2011) and proliferation (Davies et al., 1986; Metaxa et al., 2008; Sho et al., 2003). Physiological levels of shear stress, on the other hand, induce endothelial cell quiescence. Our analysis indicates that the physiological relevant signal during development is the presence of lower shear stress levels. Under pathologically high shear stress, we cannot exclude that shear stress could be proangiogenic.

Our previous results had indicated that shear stress only inhibited sprouting in veins but not in arteries (Chouinard-Pelletier et al., 2013). Similarly, others had found that steady shear stress inhibited sprouting in venous or capillary endothelial cell types (i.e. HUVECs and HMECs) 
but not in arterial endothelial cell types (BAECs) (Tressel et al., 2007). Our current results, however, indicate the opposite. Our previous results were based on an embryonic model in which we altered shear stress levels by altering the viscosity of the blood (Chouinard-Pelletier et al., 2013). Increased viscosity not only changes shear stress but would also create a more drastic pressure drop within the vascular network, such that pressure differences between vessels in the veins may be smaller. The alternative is that increasing the shear stress levels throughout the embryo resulted in an arterialization of the venous network. Though we cannot differentiate between these possibilities, it remains clear that the arterial and venous identity of the bed affects the relationship between shear stress and sprouting dynamics.

Our results also indicated that though locations where two flows merge creates points of low shear stress and large pressure differences, sprouts do not initiate from these points. We investigated the flow during the cardiac cycle to identify whether flow reversals or oscillations were present at these locations, but found that the flow remained unidirectional throughout the cardiac cycle. Whether vorticity affects endothelial cells has never been studied, but it seems unlikely since vorticity is not a force but rather a measure of the rate of rotation of the fluid elements. The endothelium is a continuous layer connected by gap junctions and endothelial cells can sense gradients in shear stress (Dolan et al., 2011; Rouleau et al., 2010). Points where there is a change in sign in the vorticity are surrounded by shear stress vectors on either side that are in opposite directions. As such, these points do not have a zero gradient in shear stress (i.e. where the magnitude and sign of shear stress is the same on both sides of the sprouting location) and do not represent true minima in shear stress.

We find the pressure difference between vessels, and not the absolute value of the relative pressure, is proportional to elongation rate. It is unlikely that the hydrostatic pressure itself is biologically active in our model. The pressure differences that we observed in these vessels are several orders of magnitude smaller than the levels of hydrostatic pressure that have been shown to be biological active (Sato and Ohashi, 2005). The pressure difference between the 
vessels does, however, control the rate at which fluids exit a vessel and therefore regulates the interstitial flow patterns. Interstitial flow alone, without a VEGF gradient, can promote sprouting in an in vitro system (Song and Munn, 2011). Interstitial flow will modify the distribution of growth factors and metabolites in the interstitial tissue, and it was previously shown that a gradient of VEGF in the presence of interstitial flow was more effective at promoting sprouting than either alone (Song and Munn, 2011). As such, rather than sprout elongation being controlled by pressure directly, current in vitro evidence would indicate that the interstitial flow is the instructive parameter. We are currently developing computational models to study the distribution of angiogenic cues and the range of interstitial flow to further investigate this phenomenon.

\section{Methods}

Simultaneous imaging of vascular remodelling and blood flow dynamics

A detailed description of the methods was previously described (Ghaffari, et al., Development, accepted). Fertilized quail eggs (Cortunix japonica) were incubated at $37^{\circ} \mathrm{C}$ and approximately $60 \%$ humidity until they reached a developmental stage of $12-14$ somites or Hamburger Hamilton 11 (HH11). The embryos were injected intravascular with labelling dyes using a picospritzer III micro-injector (General Valve Corp.). AlexaFluor 488 acetylated low-density lipoprotein (AF488-AcLDL, Invitrogen) was used to label endothelial cells and PEGylated polystyrene microspheres $(0.5 \mu \mathrm{m}$ diameter red, Invitrogen, Amino-PEG, Laysan Bio) were injected to image the flow dynamics. Embryo culture for time-lapse microscopy was done as previously described (Al-Roubaie et al., 2012). Embryos were imaged on an upright fluorescence microscope equipped with an Axiocam MRC for images of the vascular morphology (AF488-AcLDL) and a high-speed camera (Photron FASTCAM Ultima APXRS) for images of the microsphere motion. Endothelial cells were imaged with a 5x objective lens every 15 minutes. Blood flow dynamics were imaged with a 10x objective lens at $250 \mathrm{fps}$ for 2 full cardiac cycles (equivalent to 2 seconds of imaging time), once every 2 hours. 
Image processing and micro-particle image velocimetry analysis

The green image of the vasculature and the 500 red images of the microsphere motion were flattened into a single image to identify the walls of perfused vessels. A median filter was applied to the flattened image and then the image was thresholded to obtain a binary image used as the "image mask" (for additional details, see Ghaffari, et al., Development, accepted). The image mask was applied to each individual frame of the microsphere motion. The centreline of each vessel was found by skeletonizing the image mask. A Canny algorithm for edge detection was applied to the image mask to identify vessel walls. The local vessel diameter was obtained based on the vessel centreline and the vessel walls. Flow inlets and outlets were chosen far from branch points. $\mu$-PIV was used to calculate velocity waveforms based on the microsphere motion during the cardiac cycle at these inlets and outlets. Since the Reynolds number for the flow is very close to one and the Womersley number is less than one (Jones et al., 2004), entrance length effects are on the order of microns and a fully developed velocity profile was assumed for the $\mu$-PIV analysis.

\section{Computational fluid dynamics}

The vascular geometry obtained by image processing and the inlet/outlet velocities acquired by $\mu$-PIV were imported into a computational fluid dynamic (CFD) solver. The governing equations inside the vessels (i.e. continuity and momentum equations for laminar, nonNewtonian, incompressible flow) were solved using a finite elements method (FEM) in COMSOL Multiphysics 4.4. The unsteady form of the momentum equations was used for flow inside the vessels to take into account the effects of blood flow pulsatility. The viscosity of blood was adjusted for embryonic stage, vessel diameter and shear rate as previously described (Ghaffari, et al., Development, accepted). From $N$ inlets and outlets, the flow rates at $N-1$ points were set as boundary conditions. For the last outlet, the fully developed boundary condition was applied at the extended outlet of the domain (depends on Reynolds 
number), i.e. $\frac{\partial}{\partial x}=0$. Mesh generation and solution was performed by COMSOL. The domain was meshed with extremely fine free triangular meshes. Two convergence criteria were used: first, a mass flux residual of less than $10^{-8}$ for each control volume; second, $\left(\left|\varphi_{i+1}-\varphi_{i}\right|\right) /\left|\varphi_{i+1}\right| \leq 10^{-10}$ for all time steps where $\varphi$ represents $\mathrm{v}_{\mathrm{x}}$ or $\mathrm{v}_{\mathrm{y}}$ and $\mathrm{i}$ is the number of iteration.

\section{Statistical Analysis}

For formation of sprouts on the lower pressure side, a chi-squared test was used to compare the probability of sprouts always forming on one side $(n=6)$ with the probability of sprouts forming randomly ( $\mathrm{n}=3$ and $\mathrm{n}=3$, for low and high pressure side respectively). For sprout location at shear stress minima, the arc length was divided into 6 possible sprouting locations and a chi-squared test was used to evaluate the probably that sprouts would form in one location rather than randomly in all 6 locations. The value of 6 was chosen as a conservative value since it would equate approximately to $30 \mu \mathrm{m}$ of arc length. Sprouts form within microns of predicted shear stress minima. For the relationship between pressure difference and elongation rate, we tested for co-integration using a Dickey-Fuller test and established that the relationship between the variables was stationary $(\mathrm{p}<0.05)$.

\section{Sources of Funding}

EAVJ was supported by grants from the Sick Kids Foundation of Canada (NI12-029) and a grant from Life Science Research Partners. RLL was supported a grant from the Canadian Institute of Health Research (MOP-119292). SG was supported by a McGill Engineering Doctoral Awards. 


\section{Author Contributions}

SG performed experiments, developed methodology, analysed data and wrote the paper. RLL

developed methodology, analysed data and edited the paper. EAVJ performed experiments,

developed methodology, analysed data and wrote the paper.

\section{References}

Al-Roubaie, S., Hughes, J. H., Filla, M. B., Lansford, R., Lehoux, S. and Jones, E. A. (2012). Time-lapse microscopy of macrophages during embryonic vascular development. Dev Dyn 241, 1423-1431.

Bai, Y., Leng, Y., Yin, G., Pu, X., Huang, Z., Liao, X., Chen, X. and Yao, Y. (2014). Effects of combinations of BMP-2 with FGF-2 and/or VEGF on HUVECs angiogenesis in vitro and CAM angiogenesis in vivo. Cell Tissue Res 356, 109-121.

Brown, M. S., Basu, S. K., Falck, J. R., Ho, Y. K. and Goldstein, J. L. (1980). The scavenger cell pathway for lipoprotein degradation: specificity of the binding site that mediates the uptake of negatively-charged LDL by macrophages. $J$ Supramol Struct 13, 67-81.

Carmeliet, P. and Jain, R. K. (2011). Molecular mechanisms and clinical applications of angiogenesis. Nature 473, 298-307.

Chouinard-Pelletier, G., Jahnsen, E. D. and Jones, E. A. (2013). Increased shear stress inhibits angiogenesis in veins and not arteries during vascular development. Angiogenesis 16, 71-83.

Davies, P. F., Remuzzi, A., Gordon, E. J., Dewey, C. F., Jr. and Gimbrone, M. A., Jr. (1986). Turbulent fluid shear stress induces vascular endothelial cell turnover in vitro. Proc Natl Acad Sci U S A 83, 2114-2117.

Dolan, J. M., Meng, H., Singh, S., Paluch, R. and Kolega, J. (2011). High fluid shear stress and spatial shear stress gradients affect endothelial proliferation, survival, and alignment. Ann Biomed Eng 39, 1620-1631.

Galie, P. A., Nguyen, D. H., Choi, C. K., Cohen, D. M., Janmey, P. A. and Chen, C. S. (2014). Fluid shear stress threshold regulates angiogenic sprouting. Proc Natl Acad Sci U S A 111, 7968-7973.

Hallmann, R., Feinberg, R. N., Latker, C. H., Sasse, J. and Risau, W. (1987). Regression of blood vessels precedes cartilage differentiation during chick limb development. Differentiation 34, 98-105.

Hanahan, D. and Folkman, J. (1996). Patterns and emerging mechanisms of the angiogenic switch during tumorigenesis. Cell 86, 353-364.

Hobson, B. and Denekamp, J. (1984). Endothelial proliferation in tumours and normal tissues: continuous labelling studies. Br J Cancer 49, 405-413.

Jones, E. A., Baron, M. H., Fraser, S. E. and Dickinson, M. E. (2004). Measuring hemodynamic changes during mammalian development. Am J Physiol Heart Circ Physiol 287, H1561-1569.

Lampugnani, M. G. and Dejana, E. (2007). Adherens junctions in endothelial cells regulate vessel maintenance and angiogenesis. Thromb Res 120 Suppl 2, S1-6.

Lin, K., Hsu, P. P., Chen, B. P., Yuan, S., Usami, S., Shyy, J. Y., Li, Y. S. and Chien, S. (2000). Molecular mechanism of endothelial growth arrest by laminar shear stress. Proc Natl Acad Sci U S A 97, 9385-9389.

Metaxa, E., Meng, H., Kaluvala, S. R., Szymanski, M. P., Paluch, R. A. and Kolega, J. (2008). Nitric oxide-dependent stimulation of endothelial cell proliferation by sustained high flow. Am J Physiol Heart Circ Physiol 295, H736-742. 
Murakami, T., Suzuma, K., Takagi, H., Kita, M., Ohashi, H., Watanabe, D., Ojima, T., Kurimoto, M., Kimura, T., Sakamoto, A., et al. (2006). Time-lapse imaging of vitreoretinal angiogenesis originating from both quiescent and mature vessels in a novel ex vivo system. Invest Ophth Vis Sci 47, 5529-5536.

Pries, A. R. and Secomb, T. W. (2014). Making microvascular networks work: angiogenesis, remodeling, and pruning. Physiology 29, 446-455.

Rouleau, L., Rossi, J. and Leask, R. L. (2010). The Response of Human Aortic Endothelial Cells in a Stenotic Hemodynamic Environment: Effect of Duration, Magnitude, and Spatial Gradients in Wall Shear Stress. J Biomech Eng 132.

Sato, M. and Ohashi, T. (2005). Biorheological views of endothelial cell responses to mechanical stimuli. Biorheology 42, 421-441.

Sho, E., Komatsu, M., Sho, M., Nanjo, H., Singh, T. M., Xu, C., Masuda, H. and Zarins, C. K. (2003). High flow drives vascular endothelial cell proliferation during flowinduced arterial remodeling associated with the expression of vascular endothelial growth factor. Exp Mol Pathol 75, 1-11.

Siefert, S. A. and Sarkar, R. (2012). Matrix metalloproteinases in vascular physiology and disease. Vascular 20, 210-216.

Song, J. W. and Munn, L. L. (2011). Fluid forces control endothelial sprouting. Proc Natl Acad Sci U S A 108, 15342-15347.

Tah, V., Orlans, H. O., Hyer, J., Casswell, E., Din, N., Sri Shanmuganathan, V., Ramskold, L. and Pasu, S. (2015). Anti-VEGF Therapy and the Retina: An Update. J Ophthalmol 2015, 627674.

Tressel, S. L., Huang, R. P., Tomsen, N. and Jo, H. (2007). Laminar shear inhibits tubule formation and migration of endothelial cells by an angiopoietin-2-dependent mechanism. Arterioscler Thromb Vasc Biol 27, 2150-2156. 


\section{Figure Legend}

Figure 1 - Fluid dynamic analysis over a ten-hour time-lapse period. All time-lapse movies started with embryos at 12-14 somites (or Hamburger Hamilton Stage 11) and embryos were imaged for a total of 12-16 hours. The flow dynamics during sprouting angiogenesis were analysed for a total of 6 embryos, 3 in the arterial region of the capillary plexus and 3 in the venous region (A). Analysis of the time-lapse recordings was performed on data from 30 minutes before the sprout was first observed until the new vessel lumenized and flow was present in the new vessel (B, yellow arrowheads indicate sprout). Blood flow velocity and pattern was determined for the entire cardiac cycle however only values for peak systole are presented (C, D, Supplemental Movie 1). By combining viscosity estimates with the velocity profiles in the vessels, the relative pressure (Supplemental Movie 2), shear stress (Supplemental Movie 3) and vorticity (Supplemental Movie 3) were calculated. Scale bars; $1000 \mu \mathrm{m}(\mathrm{A}), 100 \mu \mathrm{m}(\mathrm{B}-\mathrm{D})$.

Figure 2 - Sprouts form from vessels at lower pressure towards vessels at higher pressure. Contour plots for the relative pressure in all 6 embryos followed by time-lapse microscopy are presented ( 3 arterial, A-B, and 3 venous, C-D). Plots represent calculated relative pressure just prior to the onset of sprouting (30 minutes before the sprout is visible, A, C). Sprout location is shown in the green image of the vascular network $(B, D)$ and indicated on contour pots by the black arrows (A, C). A positive pressure differential between the two vessels was always present, both for arteries (A) and veins (C). Scale bar: $100 \mu \mathrm{m}$.

Figure 3 - Sprouts form in a local minimum of shear stress. Contour plots show shear stress levels for a subsection of the lower pressure region in the arterial vascular network of three embryos, 30 minutes prior to the sprout being visible (A). Black lines and arrows indicate the starting location and the direction for the arc length that is plotted as the $\mathrm{x}$-axis in panel B. 
Data for the entire arclength is presented in Supplemental Fig. 1. Sprout location is indicated by a black arrow in the plot of shear stress versus arc length (B, $24 \mu \mathrm{m}$ for embryo 1, $36 \mu \mathrm{m}$ for embryo 2, $36 \mu \mathrm{m}$ for embryo 3). Sprouting location represents a local minimum in shear stress for all three embryos. Scale bar: $100 \mu \mathrm{m}$.

Figure 4-Sprouting never occurred at locations where two blood flow streams merge. Velocity plots show locations of low shear stress points (blue and orange arrows) with respect to the flow patterns present (A). The blue arrow indicates sprouting location and the orange arrow indicates a non-sprouting shear stress minimum. The non-sprouting minimum occurs at a point where streams merge and a change in sign of the vorticity is present $(\mathrm{B}$, blue to red transition in contour plot). In the first embryo, two shear stress minima are present at $22 \mu \mathrm{m}$ and $132 \mu \mathrm{m}(\mathrm{C}$, plots show half the total arc length representing the lower $50 \%$ of pressure values). The sprout forms at the first shear stress minimum ( $22 \mu \mathrm{m}$, or blue arrow in A, B). The latter shear stress minimum $(132 \mu \mathrm{m}$, black arrow in A, B) represents a point where streams merge and a change in sign in vorticity is present (D). The exclusion of sprouting from locations where vorticity is zero was consistently observed in all three embryos (E-F for Embryo 2, and G-H for Embryo 3). Dotted black lines indicate locations where vorticity is equal to zero. Scale bar: $100 \mu \mathrm{m}$.

Figure 5 - Application of these three hemodynamic parameters can predict sprouting location in perfused networks. The hemodynamic parameters for sprouting in 4 embryos were analysed blind to sprout location. Only the lowest $50 \%$ of relative pressure in each embryo were considered as possible sprouting locations (A). The base of each arrow in panel A indicates the start of the $\mathrm{X}$-axis in panel B. Locations where the vorticity is equal to zero were identified and excluded from the analysis of shear stress minima (B). The lowest remaining shear stress minimum was chosen as the predicted sprouting location. When compared with 
the actual sprouting location (C), in all four cases the sprouting location was successfully identified ( $\mathrm{n}=4$ of 4 embryos). Scale bar: $100 \mu \mathrm{m}$.

Figure 6 - The rate of elongation is proportional to the magnitude of the pressure differential. Sprouts were often observed to extend and regress (A, yellow arrows indicate direction of sprout motion). When the pressure differential was plotted with respect to time, periods of sprout regression (B) were found to occur when negative pressure differentials were present (C) in all three arterial plexuses. The rate of elongation also strongly correlated with pressure differential between the vessels (D). Plotting the data on a semi-ln plot demonstrated a logarithmic relationship between these quantities (E). Pressure differentials were normalized by $\mathrm{L}$, the distance between the two vessels. Scale bars: $100 \mu \mathrm{m}$.

Figure 7 -Sprouts will change direction to follow a positive pressure differential. A sprout can be observed extending and then changing direction over a period of one hour (A). Contour plots indicate changing pressure within the vessels during this time period (B, unlumenized sprout drawn in black). The sprout is initially extending in a direction of positive pressure differential. As the pressure differential becomes negative, the sprout regresses slightly (240 minutes) before beginning to extend in a new direction where the pressure differential is positive (C). Changes in sprouting direction were observed in 2 of 3 embryos analysed. Scale bar: $100 \mu \mathrm{m}(\mathrm{A}, \mathrm{B}, \mathrm{C})$. 

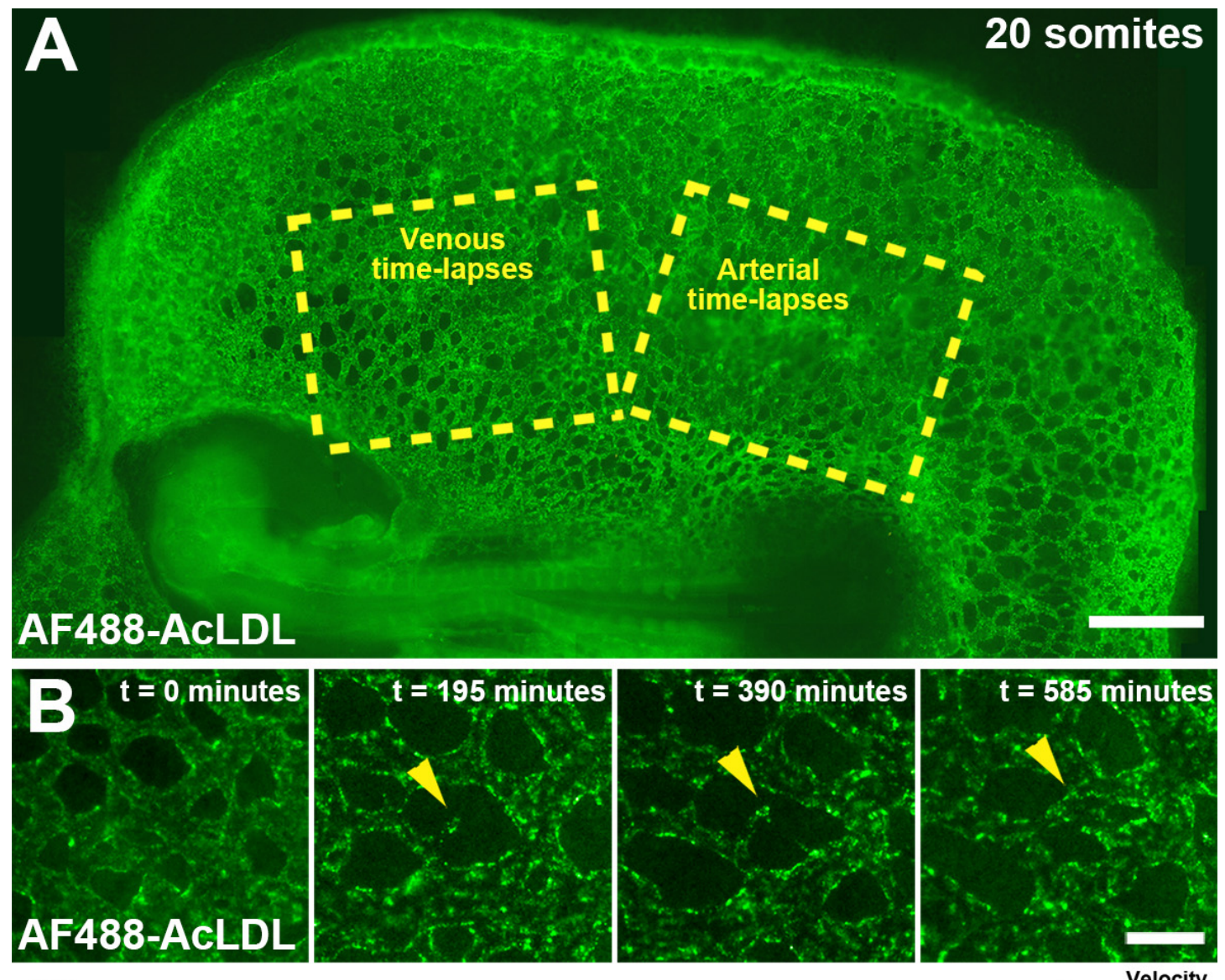

C
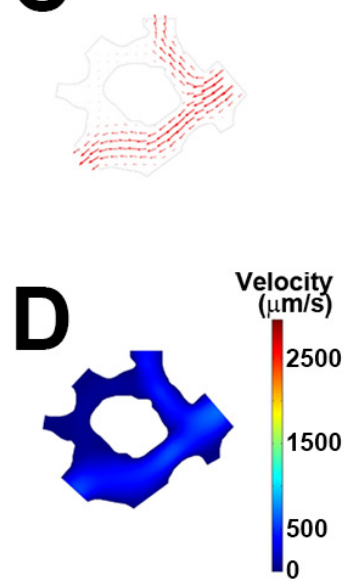
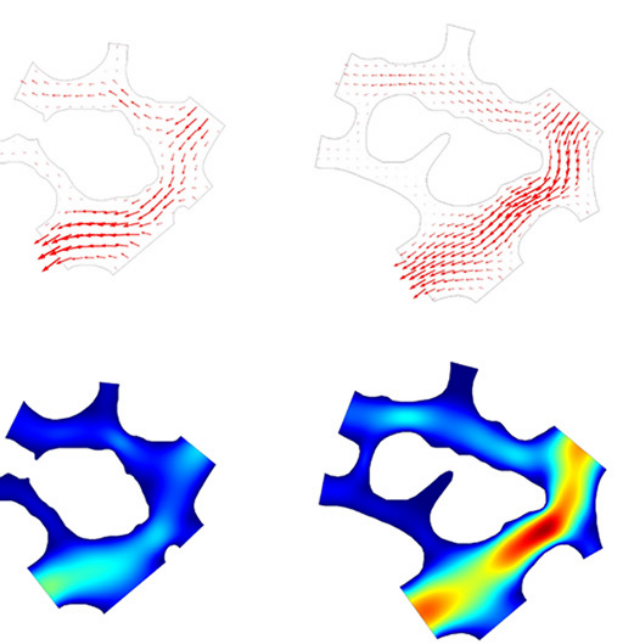

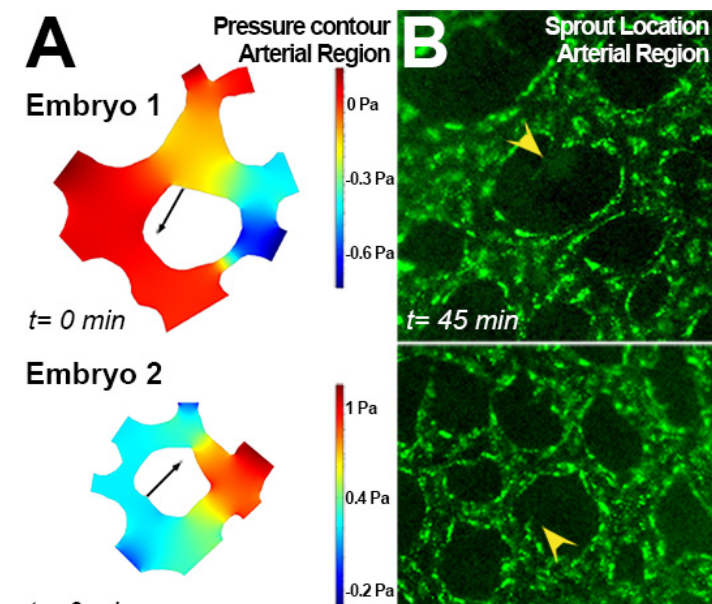

$t=0 \mathrm{~min}$

Embryo 3
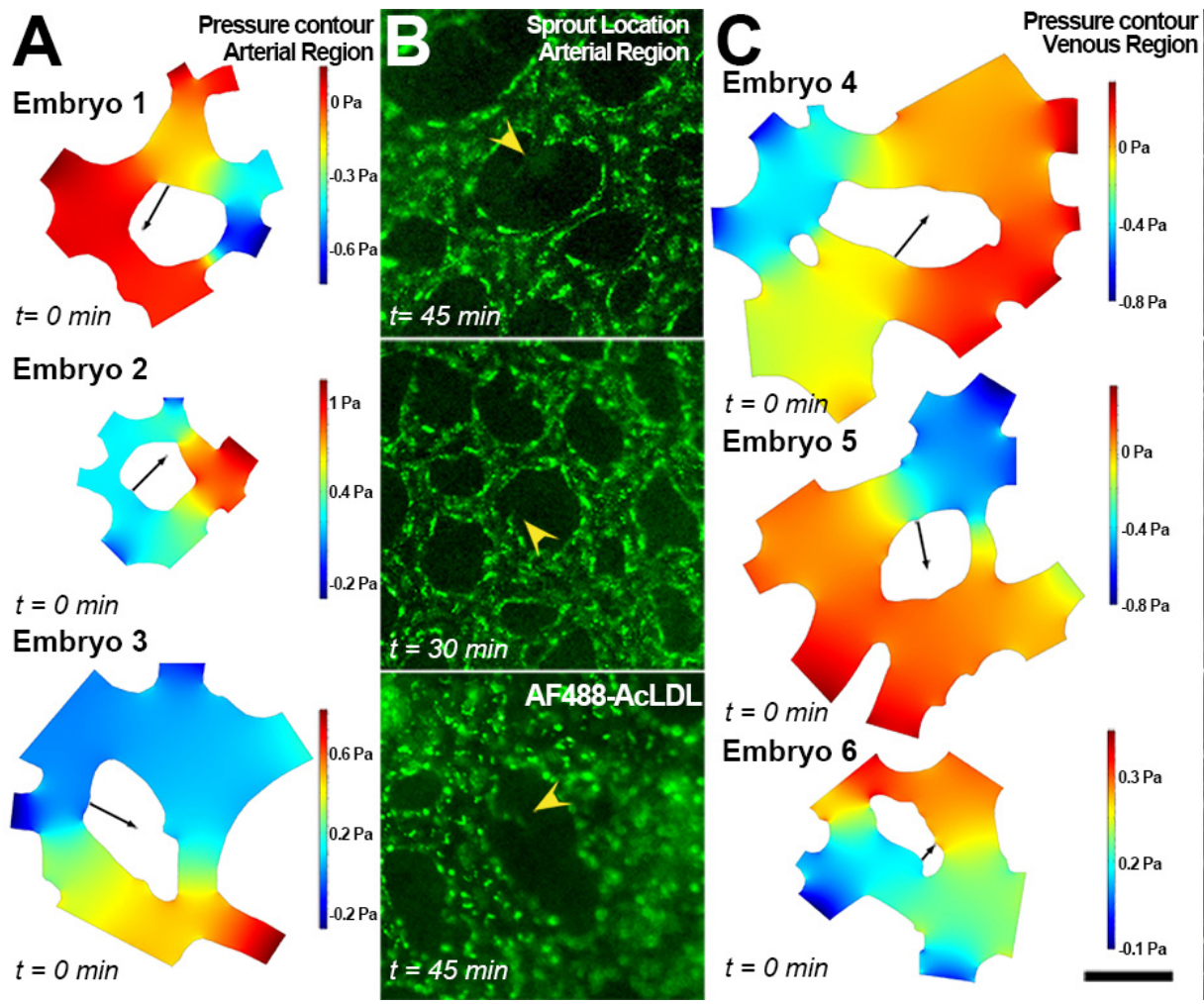

a
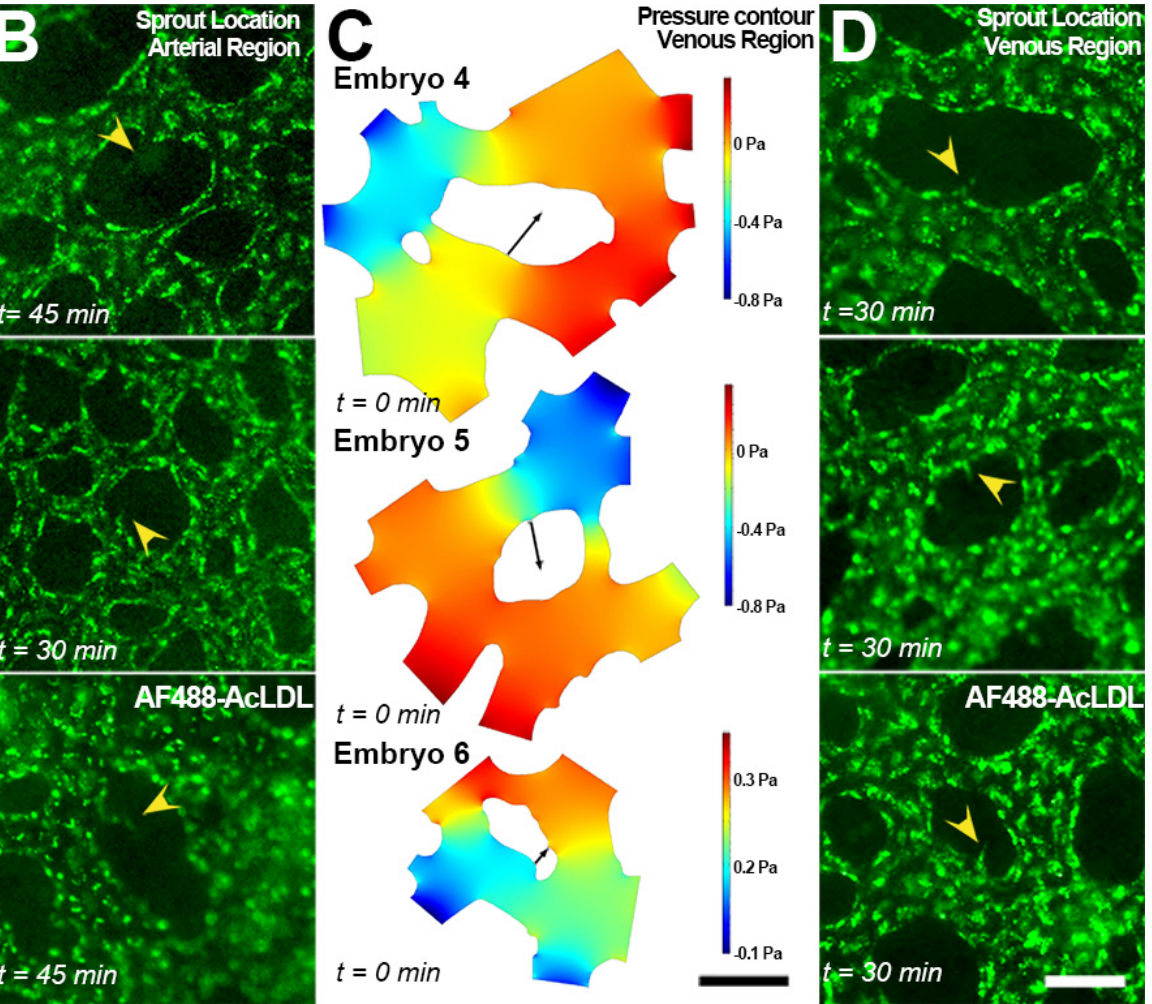

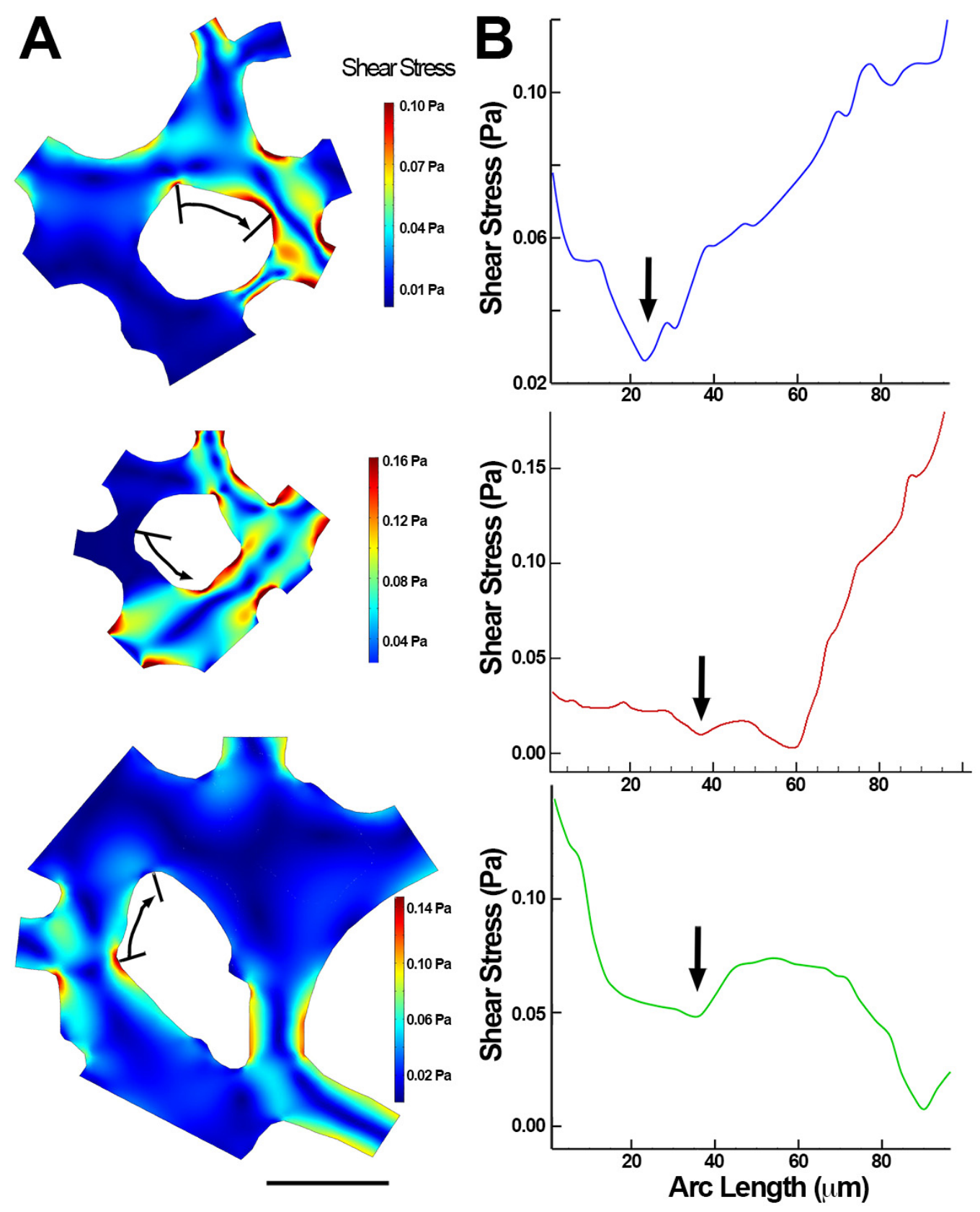

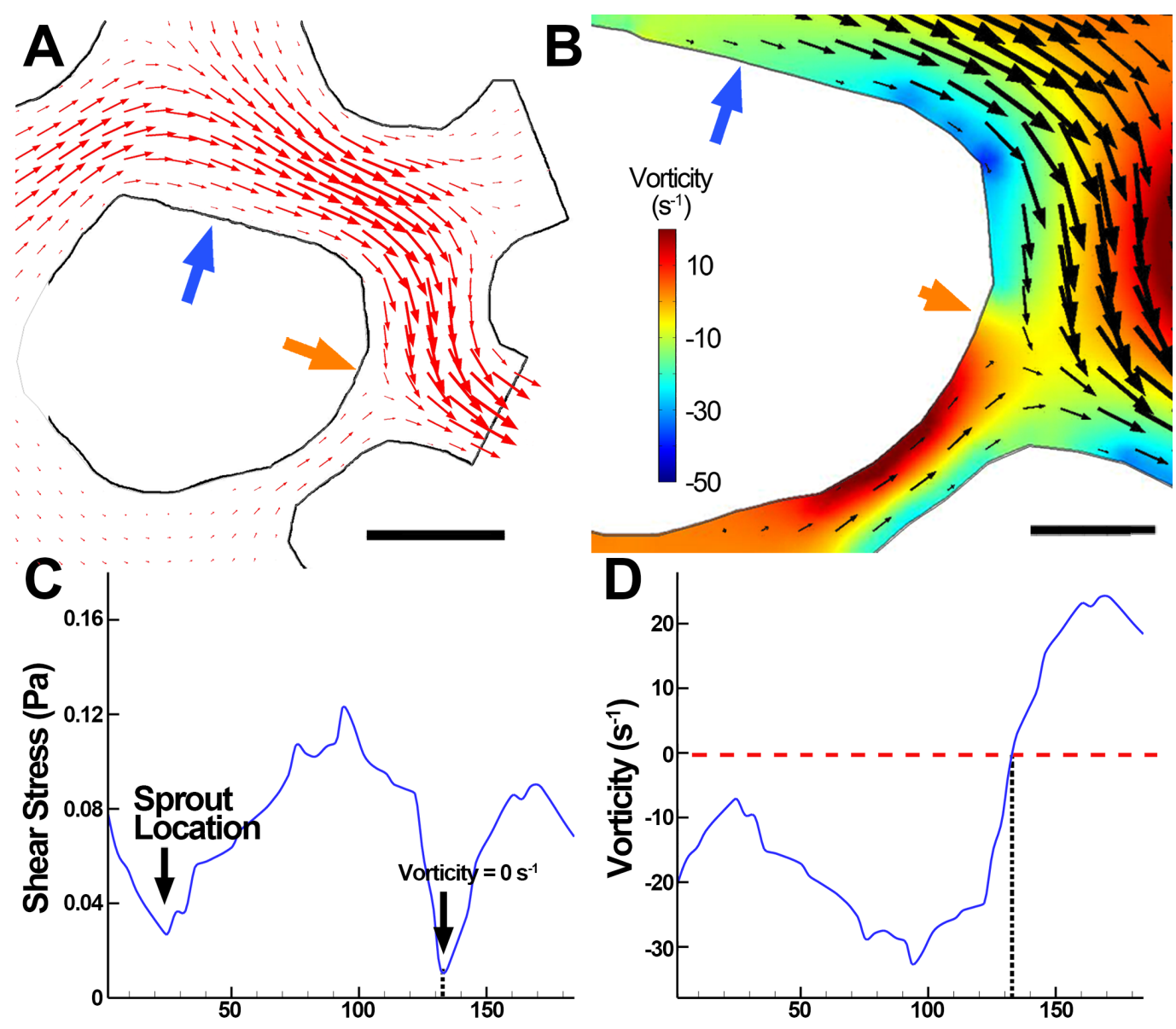

E

F
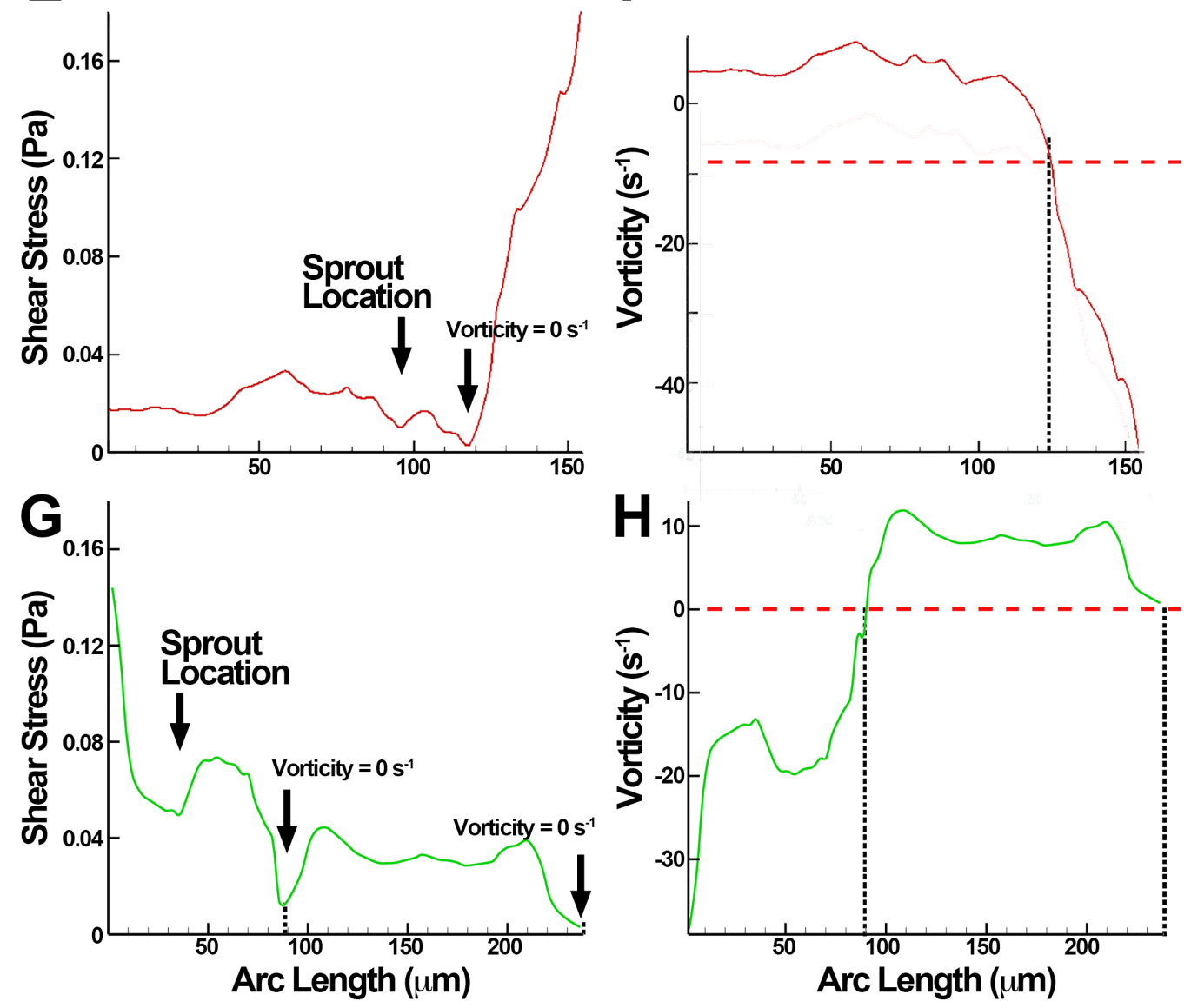

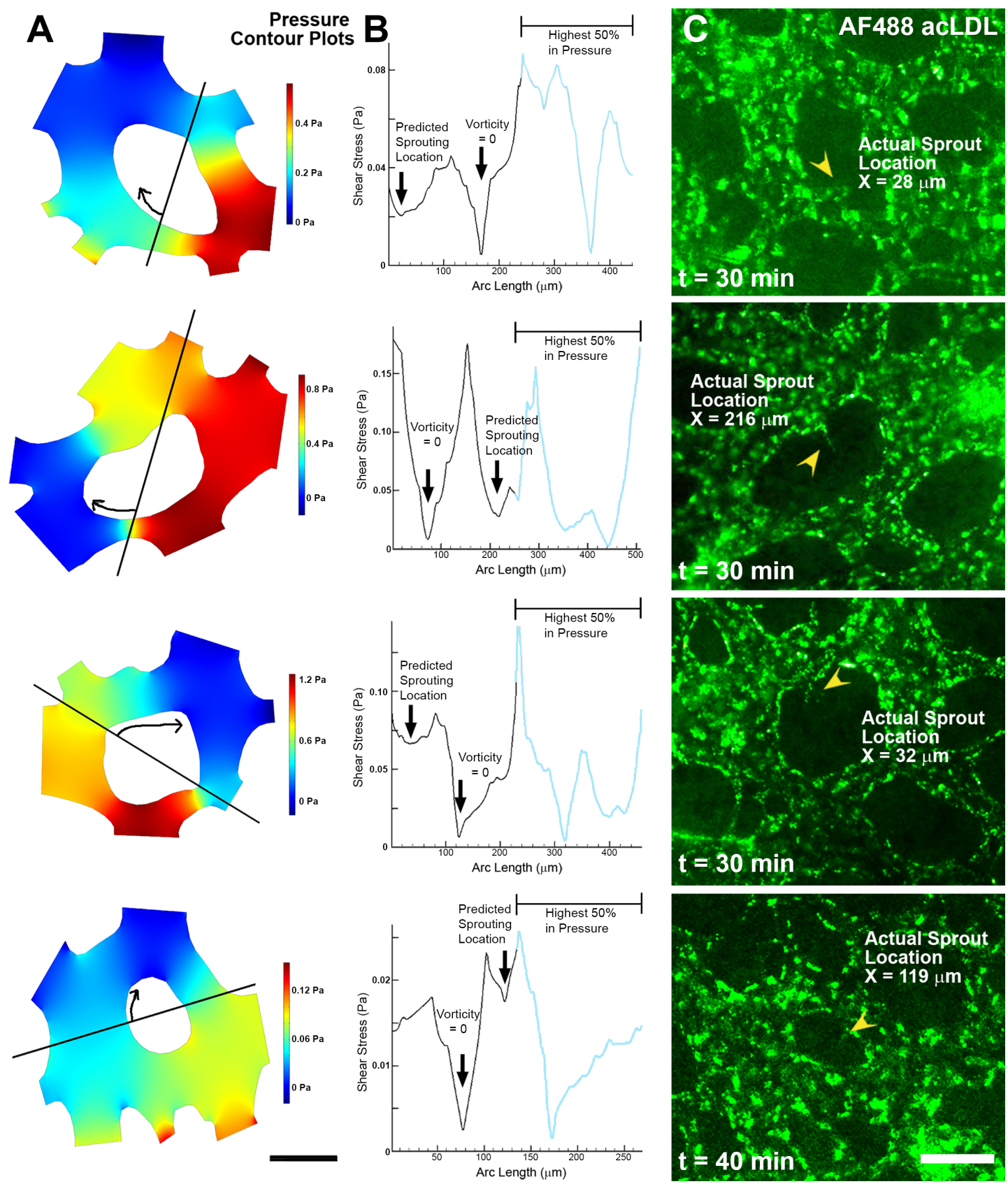


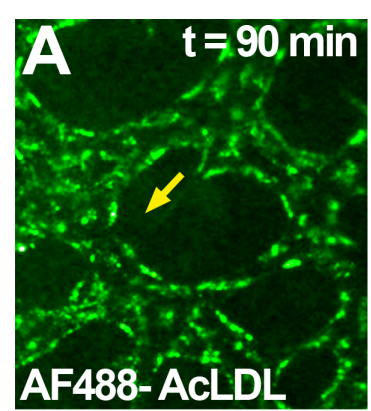

B
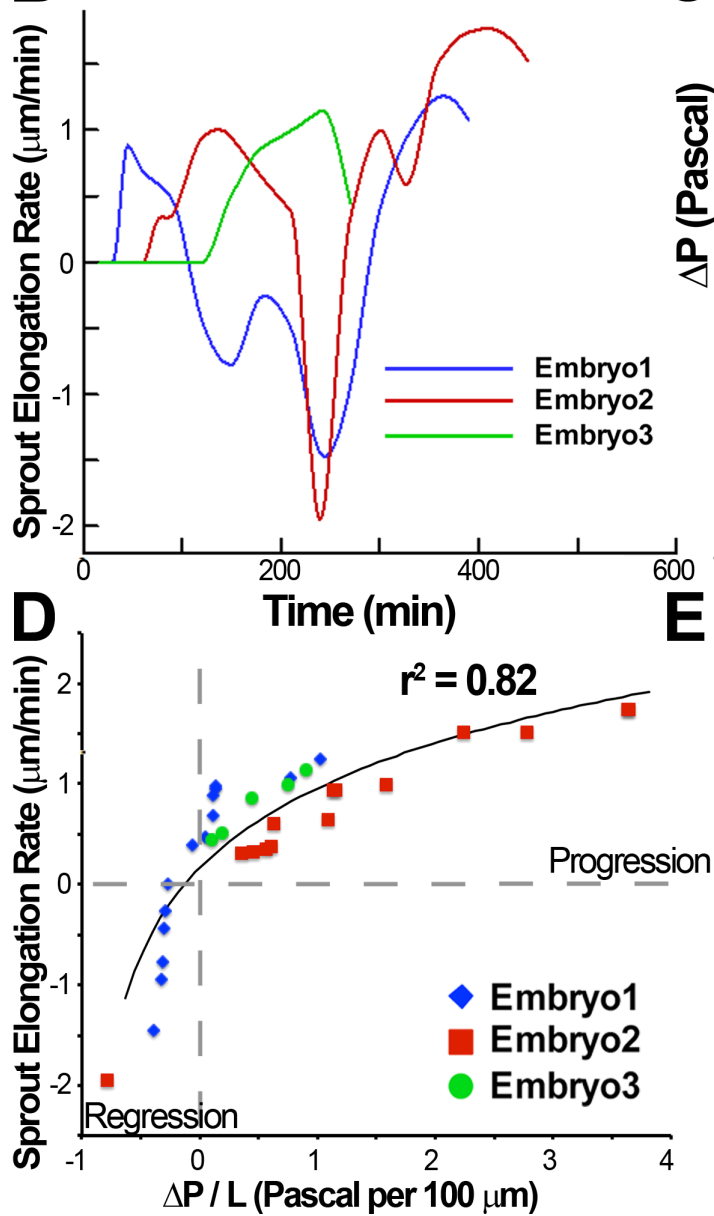
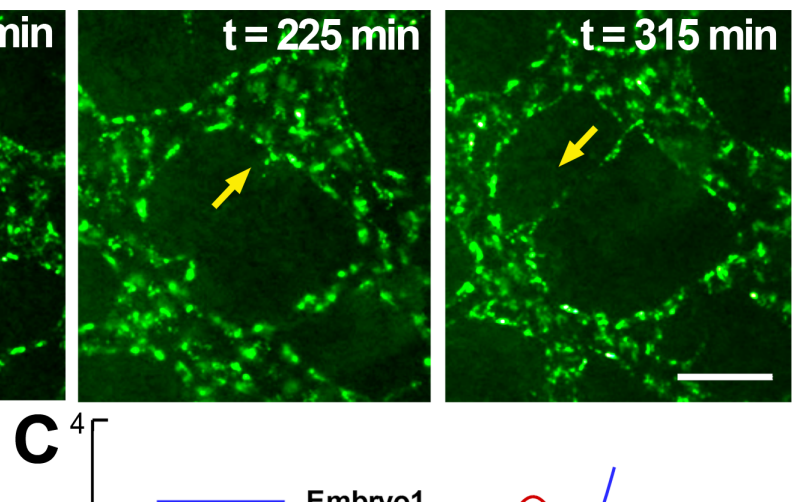

$\mathbf{C}^{4}$

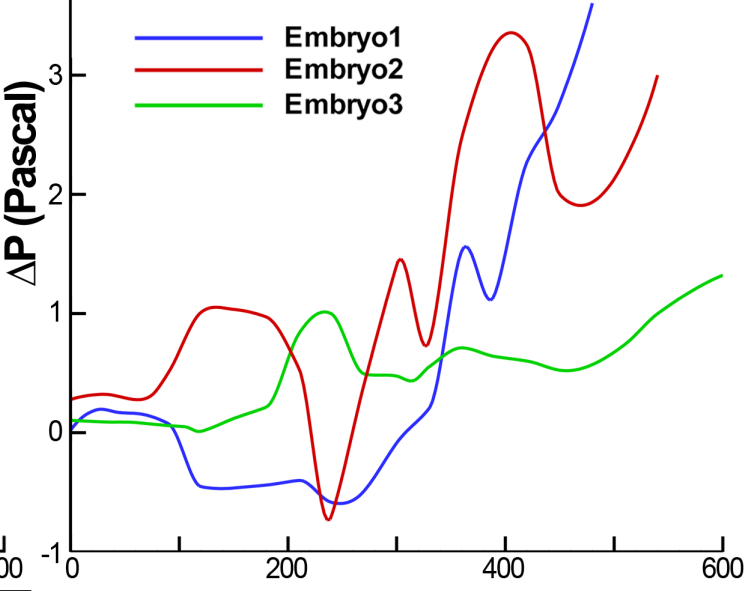
Time (min)

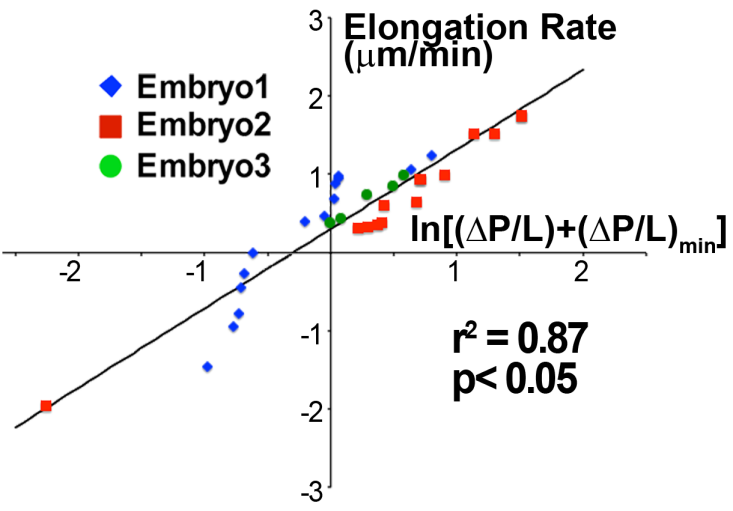



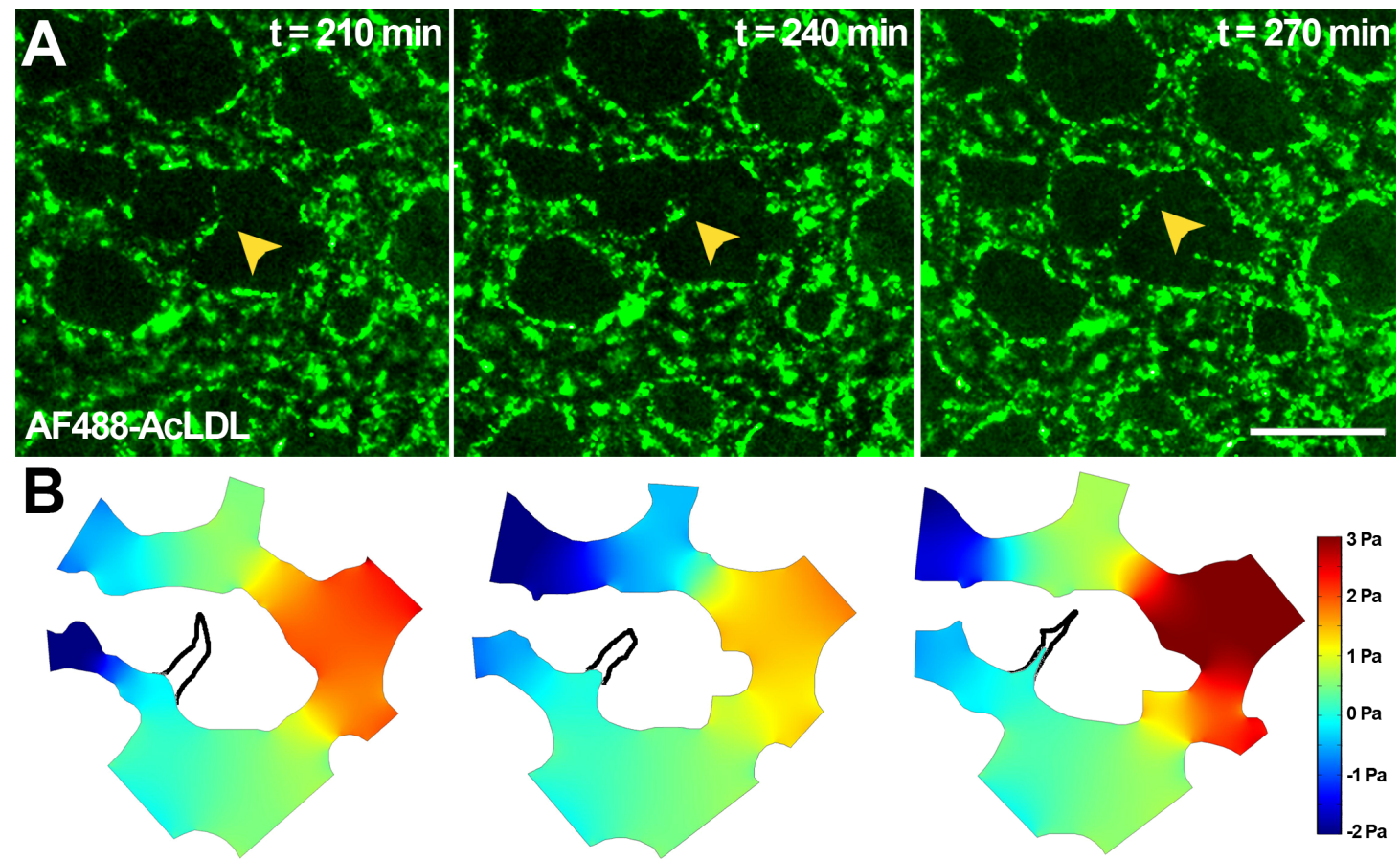

Pressure Contour Plots
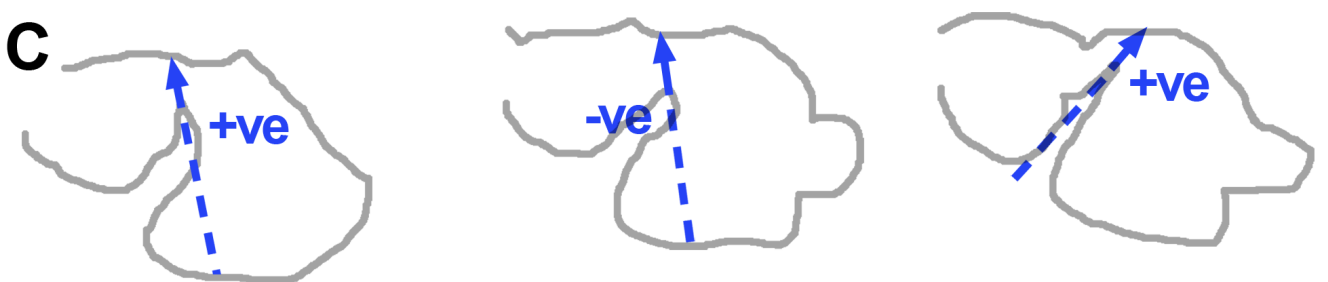

Pressure Differential in Direction of Elongation 

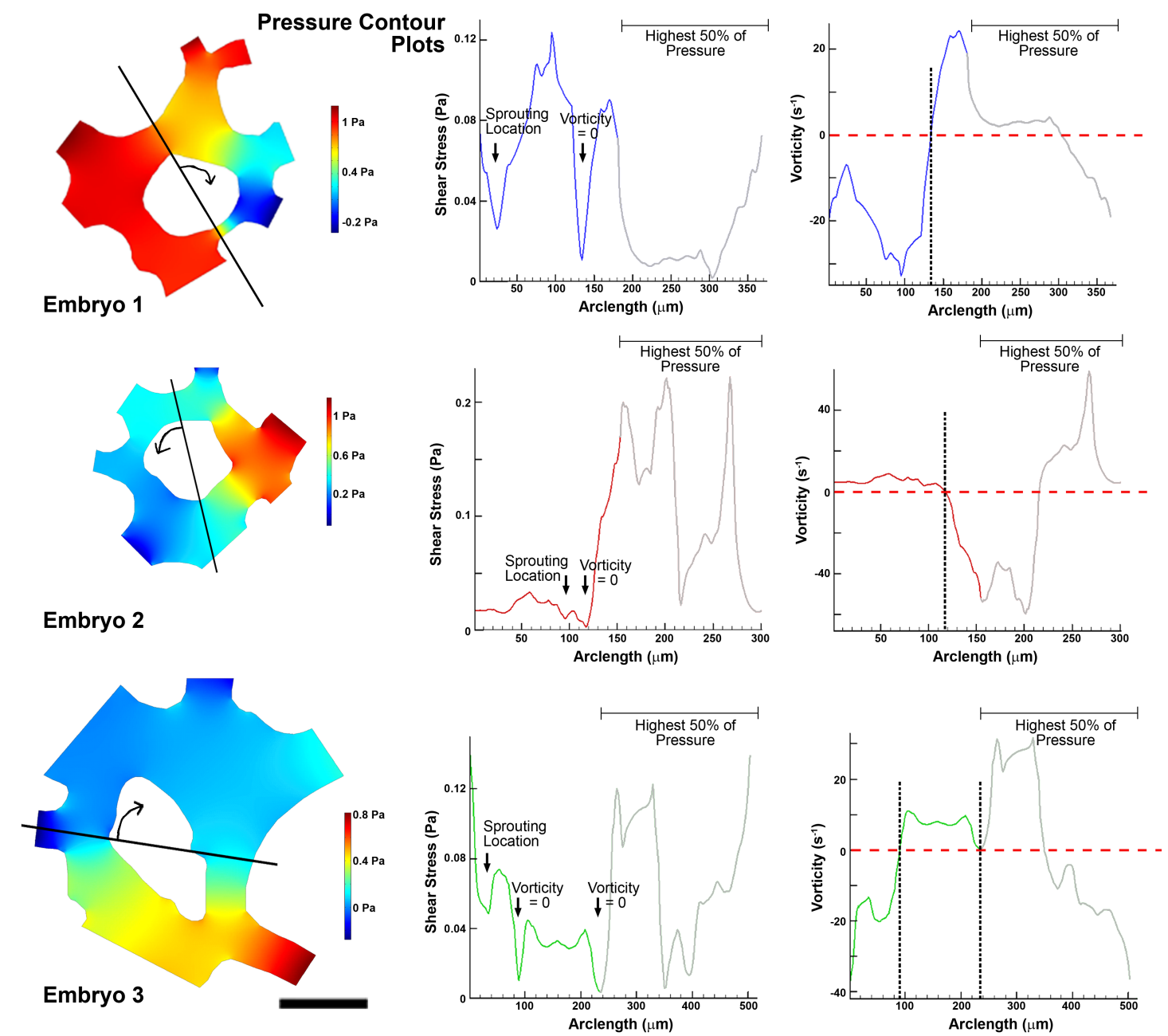

Supplemental Figure 1 - Hemodynamic data for entire arclength at the time point 30 minutes before sprout is visible. Pressure contours for each embryo are presented. Shear stress and vorticity values for the entire contour are shown, with the region at higher pressure indicated by a grey line rather than a coloured line. 


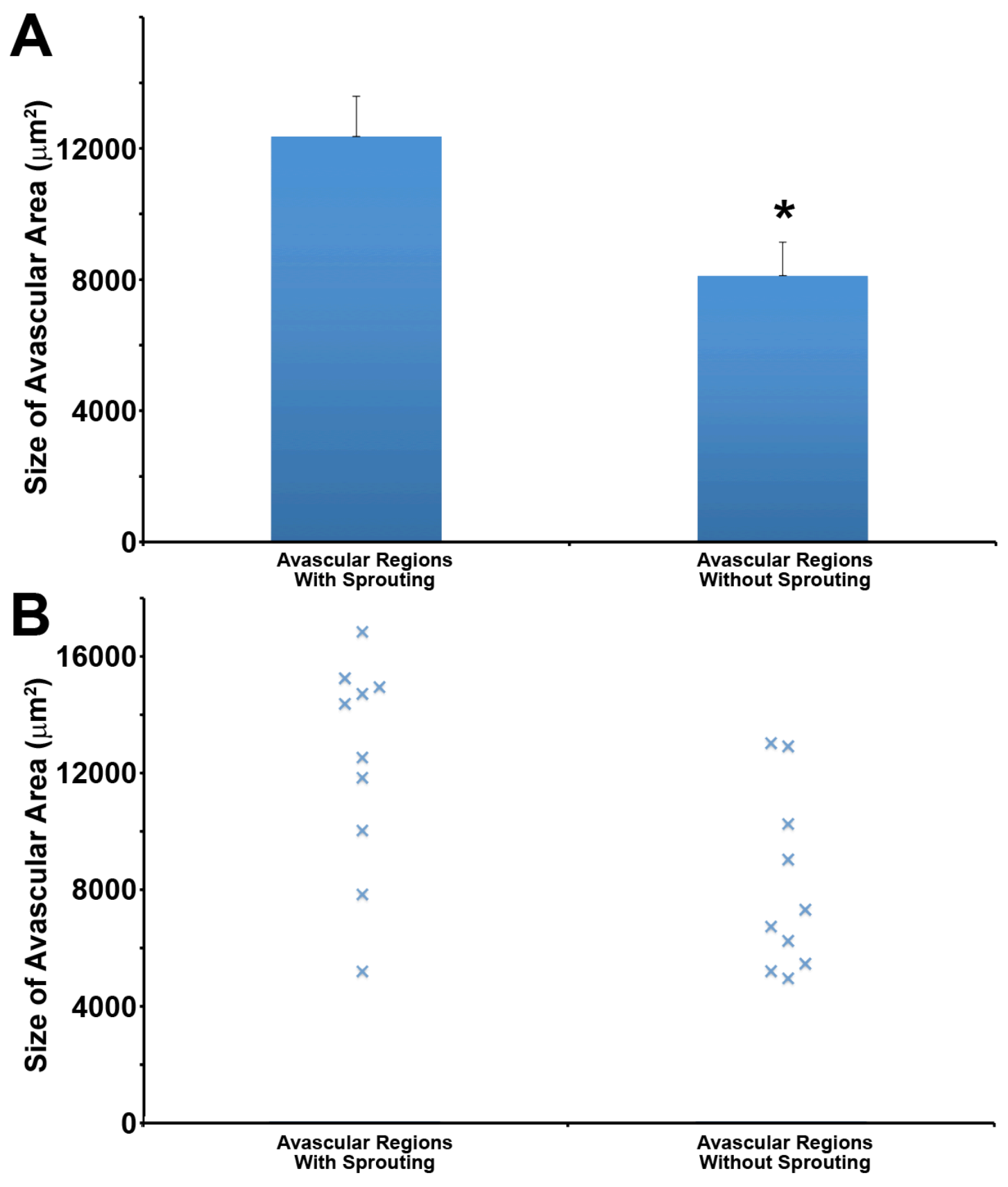

Supplemental Figure 2 - Area of avascular region is larger in sprouting than non-sprouting

location. The area of avascular regions at the time of sprouting was calculated, and compared to the area of avascular regions, at the same time point, that never sprouted during the entire time-lapse recording (A). Though sprouting avascular regions were larger than non-sprouting region $(\mathrm{p}<0.05)$, size of the region alone cannot predict which avascular regions sprout due to the large spread of the data. 


\section{Supplementary Movie Legends}

Supplemental Movie 1 - Blood velocity analysis around sprouting angiogenesis. Results of the analysis of blood velocity changes during sprouting are presented. The left panel shows the endothelial cell behaviour during the time period of analysis. The centre panel shows the velocity vectors for the blood flow during peak systole in the network. Velocity vectors are sized based on the maximum velocity at that specific time point (i.e. scale is relative). The right panel shows the absolute velocity magnitude during peak systole in these vessels during angiogenic sprouting. All scale bars represent $100 \mu \mathrm{m}$.

Supplemental Movie 2 - Pressure changes during sprouting angiogenesis. Results of the pressure calculations during sprouting are presented. The left panel shows the endothelial cell behaviour during the time period of analysis. The centre panel shows the relative pressure, where red represents the highest pressure at that specific time point. The right panel shows the same data, but the colour scale remains constant throughout all time points. All presented values are for peak systole however blood flow dynamics were analysed for the entire cardiac cycle at each time point. All scale bars represent $100 \mu \mathrm{m}$.

Supplemental Movie 3 - Shear stress and vorticity analysis around sprouting angiogenesis. Results of the shear stress and vorticity calculations at peak systole during sprouting are presented. The left panel shows the endothelial cell behaviour during the period of analysis. The centre panel shows the results for the shear stress calculations, with a constant colour scale for all time points. The right panel shows the results for the vorticity calculations, with a constant colour scale for all time points. All scale bars represent $100 \mu \mathrm{m}$. 